\title{
Study on Characteristics of Coupling Field from Super-low Altitude Target under Diverse Terrestrial Environments in Semi- active Radar System
}

\author{
Gao Xiang Zou ${ }^{1}$, Chuang Ming Tong ${ }^{1}$, Qian $\mathrm{Gao}^{2}$, Tong Wang ${ }^{1}$, Peng Peng ${ }^{1}$, Jun Chao Yuan ${ }^{3}$, Xiao Jun Jiang ${ }^{4}$, and Xin \\ Wang $^{4}$ \\ ${ }^{1}$ Air Force Engineering University, Xi'an, Shaanxi, China \\ ${ }^{2}$ Air Force Communication NCO Academy, Dalian, Liaoning, China \\ ${ }^{3}$ The 95668 Army of The Chinese People's Liberation Army, Kunming, Yunnan, China \\ ${ }^{4}$ The 95876 Army of The Chinese People's Liberation Army, Zhangye, Gansu, China
}

\begin{abstract}
A novel electromagnetic scattering model is presented for studying coupling field of super-low altitude target under diverse terrestrial environments in semi-active radar system. The PO+MEC hybrid method is applied to obtain the electromagnetic scattering of target. The Physical Optics (PO) is introduced to calculate the scattering from facets of target. The Method of Equivalent Currents (MEC) is introduced to calculate the scattering from edges of target. Antenna radiation pattern of seeker is introduced to modify traditional Four Path Method (FPM) in semi-active radar system. Based on the modified FPM, the characteristics of coupling field from super-low altitude target under diverse terrestrial environments in semi-active radar system are studied. Simulation results can use for reference in the fields of remote sensing and detection for super-low altitude target.
\end{abstract}

\section{INTRODUCTION}

Nowadays, the detection for super-low altitude target becomes a difficult global problem ${ }^{[1][2]}$. The scattering problem of target above environment becomes an important subject in the field of computational electromagnetism ${ }^{[3][4]}$. The multipath interference in the detection for super-low altitude target is usually described by coupling field. The anti-multipath method can be concluded through analyzing the characteristics of coupling field.

In this paper, a novel electromagnetic scattering model is presented for studying coupling field of superlow altitude target under diverse terrestrial environments in semi-active radar system. The PO+MEC hybrid method is used to calculate the scattering from target, and Four Path Method ${ }^{[5][6]}$ (FPM) modified by antenna radiation pattern of seeker is applied to describe the multipath effect between target and environment. From the numerical results, the anti-multipath method can be concluded.

\section{MODIFIED FOUR PATH METHOD}

Traditional Four Path Method usually simplifies multiple coupling between target and environment as interaction of field in specular direction. Therefore, multiple coupling between target and environment can be simplified as interaction between target and infinite plate.

However, traditional four path method is a very simplified model and it cannot reflect actual detection process. Actually, the antenna beam of real seeker has a certain width. If the multipath interference enters the seeker through the main lobe directly, its influences are more serious than which enters the seeker through the side lobe. Hence, we introduce antenna radiation pattern to modify the traditional Four Path Method.

Actual antenna radiation pattern is complex. In this paper, the Gauss antenna radiation pattern is introduced to simulate the seeker's antenna radiation pattern. We set one main lobe and two side lobes on both sides which can meet complexity and precision. Compared to the main lobe's gain, the side lobe's gain is small. Therefore, set the side lobe's gain as $g_{3}$.

The function of Gauss antenna radiation pattern is taken in the form:

\footnotetext{
* Corresponding author: gxiangzou@163.com, cmtong156@126.com, gaoqian717@163.com, tong_wang001@163.com, pengpengxidian@163.com, 17791252698@163.com,

994053660@qq.com, 1145115936@qq.com
} 


$$
G_{M B}(\theta)=\exp \left(-2 \ln 2 \theta^{2} / \theta_{B}^{2}\right),|\theta| \leq \mu
$$

Here $\theta_{B}$ is half-power beam point width; $\mu$ is azimuth angle when the main lobe's gain is $g_{3} . \mu$ is taken in the form:

$$
\mu=\theta_{B} \sqrt{\ln g_{3} /(-2 \ln 2)}
$$

The functions of antenna radiation pattern for the first and the second side lobe are taken in the forms:

$$
\begin{gathered}
G_{B 1}(\theta)=g_{1} \exp \left(\frac{-2 \ln 2(\theta+1.5 \mu)^{2}}{\theta_{B 1}^{2}}\right), \mu \leq|\theta| \leq 2 \mu \\
G_{B 2}(\theta)=g_{2} \exp \left(\frac{-2 \ln 2(\theta \pm 2.5 \mu)^{2}}{\theta_{B 2}^{2}}\right), 2 \mu<|\theta| \leq 3 \mu
\end{gathered}
$$

In this paper, set $g_{1}, g_{2}$ and $g_{3}$ as $0.01,10^{-2.5}$ and 0.001 . The model of modified four path method is as shown in Fig.1.

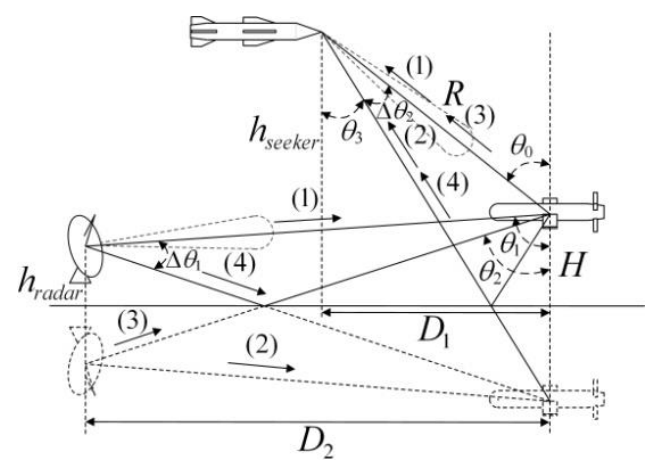

Fig.1 Model of modified four path method

As shown in Fig. $1, H$ is the height of target; $h_{\text {seeker }}$ is the height of seeker; $h_{\text {radar }}$ is the height of illuminating radar; $\mathrm{R}$ is the distance between target and seeker; $D_{1}$ is the projector distance between target and seeker; $D_{2}$ is the projector distance between target and radar; $\theta_{0}$ is the pitch angle that the seeker is relative to the target. These parameters have the relationship as follow:

$$
\left\{\begin{array}{l}
D_{1}=R \sin \theta_{i} \\
h_{\text {seeker }}=H+R \cos \theta_{i} \\
\theta_{1}=\tan ^{-1}\left[D_{2} /\left(H-h_{\text {radar }}\right)\right] \\
\theta_{2}=\tan ^{-1}\left[D_{2} /\left(H+h_{\text {radar }}\right)\right] \\
\theta_{3}=\tan ^{-1}\left[D_{1} /\left(H+h_{\text {seeker }}\right)\right]
\end{array}\right.
$$

Modified complex reflection coefficient is introduced to describe the scattering of terrestrial environment with certain roughness, which is taken in the form:

$$
\rho\left(\theta_{i}^{\text {sur }}\right)=\Gamma\left(\theta_{i}^{\text {sur }}\right) \rho_{\mathrm{s}}\left(\theta_{i}^{\text {sur }}\right)
$$

Here $\theta_{i}^{\text {sur }}$ is the incident angle where incident wave illuminates the environment; $\Gamma$ is the Fresnel reflection coefficient, and the polarization mode is Vertical-toVertical (VV) in this paper; $\rho_{\mathrm{s}}$ is the reflection factor of rough surface.

Set $\boldsymbol{E}\left(\theta_{i}, \theta_{s}\right)$ and $\boldsymbol{E}^{\prime}\left(\theta_{i}, \theta_{s}\right)$ as scattering field from target and mirror target respectively. As shown in Fig.1, the four paths can be described as follow:

Path 1: the propagation path of electromagnetic wave is 'radar $\rightarrow$ target $\rightarrow$ seeker'. The scattering field is $\boldsymbol{E}_{1}\left(\pi-\theta_{1}, \theta_{0}\right)$.
Path 2: the propagation path of electromagnetic wave is 'radar $\rightarrow$ target $\rightarrow$ environment $\rightarrow$ seeker'. The scattering field is $\rho\left(\theta_{3}\right) \boldsymbol{E}_{2}^{\prime}\left(\theta_{1}, \theta_{3}\right)$.

Path 3: the propagation path of electromagnetic wave is 'radar $\rightarrow$ environment $\rightarrow$ target $\rightarrow$ seeker'. The scattering field is $\rho\left(\theta_{2}\right) \boldsymbol{E}_{3}\left(\pi-\theta_{2}, \theta_{0}\right)$.

Path 4: the propagation path of electromagnetic wave is 'radar $\rightarrow$ environment $\rightarrow$ target $\rightarrow$ environment $\rightarrow$ seeker'. The scattering field is $\rho\left(\theta_{2}\right) \rho\left(\theta_{3}\right) \boldsymbol{E}_{4}^{\prime}\left(\theta_{2}, \theta_{3}\right)$.

As shown in Fig.1, two conditions cannot be neglected: one is that the radar wave illuminates the environment along the side lobe, and the other is that the multipath interference enters the seeker from the side lobe. Therefore, the radar's and the seeker's antenna radiation pattern must be considered. The angle between the direction of direct echo and multipath echo is $\Delta \theta$ ( $\Delta \theta_{1}$ denotes the angle between direct illuminating direction and multipath direction; $\Delta \theta_{2}$ denotes the angle between direct receiving direction and multipath direction). Set the correction factor of antenna radiation pattern as $\xi(\Delta \theta)$. The antenna gain in the direction of the main lobe's maximum gain is $\left.G_{M B}(\theta)\right|_{\theta=0^{\circ}}=1$. The correction factor is taken in the form:

$$
\xi(\Delta \theta)=\frac{G(\Delta \theta)}{\left.G_{M B}(\theta)\right|_{\theta=0^{\circ}}}=\left\{\begin{array}{l}
G_{M B}(\Delta \theta),|\Delta \theta| \leq \mu \\
G_{B 1}(\Delta \theta), \mu \leq|\Delta \theta| \leq 2 \mu \\
G_{B 2}(\Delta \theta), 2 \mu \leq|\Delta \theta| \leq 3 \mu
\end{array}\right.
$$

Based on the correction factor above, the modified Four Path Method is obtained as follow:

$$
\begin{aligned}
\boldsymbol{E}^{s}= & \boldsymbol{E}_{1}\left(\pi-\theta_{1}, \theta_{0}\right)+\xi\left(\Delta \theta_{2}\right) \rho\left(\theta_{3}\right) \boldsymbol{E}_{2}^{\prime}\left(\theta_{1}, \theta_{3}\right)+ \\
& \xi\left(\Delta \theta_{1}\right) \rho\left(\theta_{2}\right) \boldsymbol{E}_{3}\left(\pi-\theta_{2}, \theta_{0}\right)+ \\
& \xi\left(\Delta \theta_{1}\right) \xi\left(\Delta \theta_{2}\right) \rho\left(\theta_{2}\right) \rho\left(\theta_{3}\right) \boldsymbol{E}_{4}^{\prime}\left(\theta_{2}, \theta_{3}\right)
\end{aligned}
$$

Here $\boldsymbol{E}^{s}$ denotes the electric field vector which the seeker receives.

\section{FORMULATION}

\subsection{PHYSICAL OPTICS (PO)}

Super-low altitude target can be simulated by triangle facets. The far field of every triangle facet in target model can be calculated by physical optics.

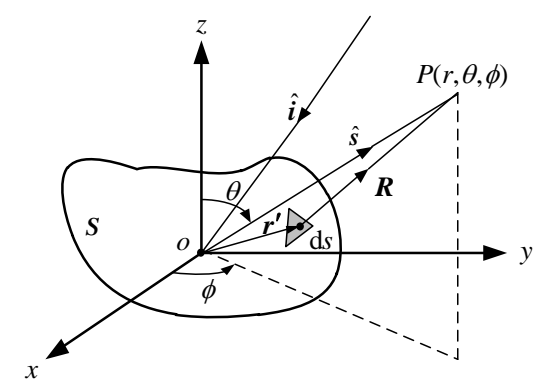

Fig.2 The electromagnetic scattering from the facet of target model 
The far field of every illuminated facet is taken in the form:

$$
\boldsymbol{E}^{\text {facet }} \approx \frac{i k_{0}}{4 \pi} \frac{e^{-j k_{0} r}}{r}\left[\hat{\boldsymbol{s}} \times\left(\boldsymbol{M}+\eta_{0} \hat{\boldsymbol{s}} \times \boldsymbol{J}\right)\right] \cdot \Delta \mathrm{A} \cdot I
$$

Detailed descriptions of the individual terms in (9) can be found in [7].

\subsection{METHOD OF EQUIVALENT CURRENTS (MEC)}

Super-low altitude target has the edge construction. The scattering from the edge construction cannot be neglected.

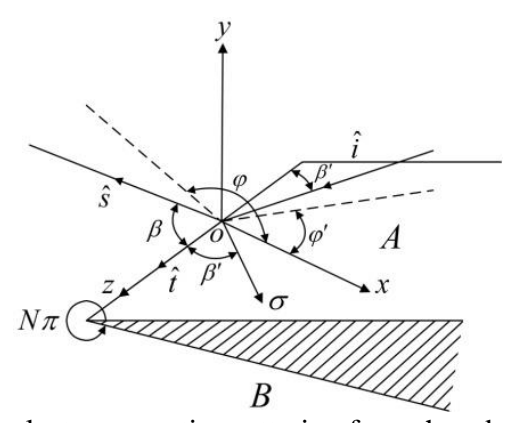

Fig.3 The electromagnetic scattering from the edge of target model

The scattering field of edge can be obtained by substituting equivalent electric/magnetic current into the radiation integral, which is taken in the form:

$$
\boldsymbol{E}^{e d g e}=\frac{i k_{0}}{4 \pi} \frac{e^{-j k_{0} r}}{r} \int_{C}\left[\eta_{0} \hat{\boldsymbol{s}} \times(\hat{\boldsymbol{s}} \times \boldsymbol{J})+\hat{\boldsymbol{s}} \times \boldsymbol{M}\right] e^{i k_{0} \hat{s} \cdot \mathbf{r}^{\prime}} d l
$$

Detailed descriptions of the individual terms in (10) can be found in [8].

\section{NUMERICAL RESULTS}

In this section, the characteristics of coupling field from super-low altitude target above diverse terrestrial environments is analyzed by modified FPM and PO+MEC hybrid method.

The power ratio is defined as the ratio of back scattering to multipath scattering (coupling field) in a certain incident direction $\left(\theta_{i}, \varphi_{i}\right)$. The power ratio is taken in the form:

$$
T / M=\frac{\left|E^{T}\left(\theta_{i}, \varphi_{i}\right)\right|^{2}}{\left|E^{M}\left(\theta_{i}, \varphi_{i}\right)\right|^{2}}
$$

Here $\left|E^{T}\left(\theta_{i}, \varphi_{i}\right)\right|^{2}$ denotes the power of back scattering from target in the direction of $\left(\theta_{i}, \varphi_{i}\right) ;\left|E^{M}\left(\theta_{i}, \varphi_{i}\right)\right|^{2}$ denotes the power of multipath scattering (coupling field) in the direction of $\left(\theta_{i}, \varphi_{i}\right)$.

Set working frequency as $10 \mathrm{GHz}$; The complex reflection coefficients of different terrestrial environments are given in Table. 1 as follow:
Table 1: Complex dielectric constants of different terrestrial environments

\begin{tabular}{cc}
\hline Environments & Complex dielectric constant \\
\hline Concrete & $3.0000-\mathrm{j} 0.000036$ \\
Dry land & $4.0000-\mathrm{j} 0.000018$ \\
Half-dry land & $7.0000-\mathrm{j} 0.072$ \\
Wet land & $30.0000-\mathrm{j} 0.36$ \\
\hline
\end{tabular}

For the following computation examples, set the target as a perfect conductor missile, and the simulation condition is the stable tracking phase in semi-active radar system; Polarization mode is VV polarization; Height of illuminating radar is $3 \mathrm{~m}$; Height of missile is $15 \mathrm{~m}$; Distance between seeker and target is $5 \mathrm{~km}$.; Horizontal projection distance between illuminating radar and target is $5 \mathrm{~km}$; Half-power beam widths of seeker and radar are $14^{\circ}$ and $0.8^{\circ}$ respectively; The observation angle ranges from $0^{\circ}$ to $90^{\circ}$ (corresponding grazing angle ranges from $90^{\circ}$ to $0^{\circ}$ ).

Modified FPM and PO+MEC hybrid method are used to calculate the bi-static scattering coefficient and to extract coupling field. Based on the target scattering field and coupling field, the curve of power ratio is obtained.

As shown in Fig.4, when target is above the concrete/dry land/half-dry land/wet land surface, the curve of coupling field arrives minimum at the Brewster angle $30^{\circ} / 25^{\circ} / 20^{\circ} / 10^{\circ}$. The results show that the multipath interference is smallest at the Brewster angle of the certain environment when the seeker detects the super-low altitude target.

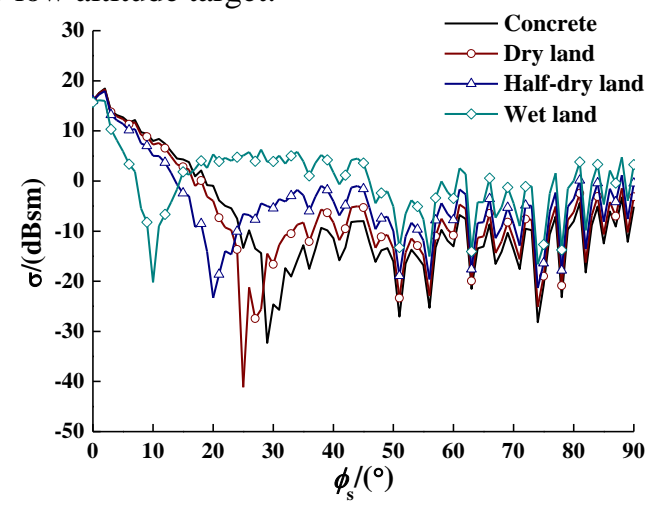

Fig.4 The coupling field of super-low altitude target under four

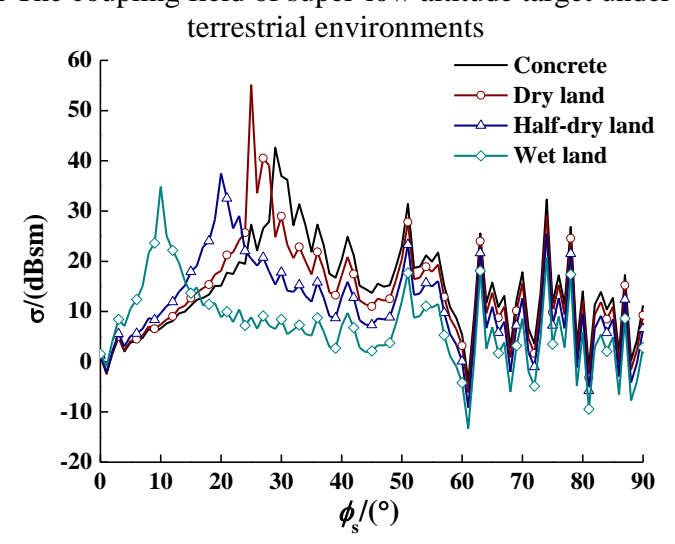

Fig.5 The power ratio of super-low altitude target under four terrestrial environments

As shown in Fig.5, when target is above the concrete/dry land/half-dry land/wet land surface, the curve of power ratio arrives maximum at the Brewster angle $30^{\circ} / 25^{\circ} / 20^{\circ} / 10^{\circ}$. The reason leads to the 
phenomenon is that the multipath interference is smallest at the Brewster angle of the certain environment when the seeker detects the super-low altitude target. The results are consistent with the results of Fig.4. Therefore, if the seeker detects super-low altitude target at the Brewster angle, the target echo will be most obvious and the multipath interference will be weakest.

\section{CONCLUSIONS}

In this paper, a novel electromagnetic scattering model is presented for studying coupling field of super-low altitude target under diverse terrestrial environments in semi-active radar system. The Gauss antenna radiation pattern is introduced to modify the Four Path Method. The PO+MEC hybrid method is applied to obtain the electromagnetic scattering of target. The curves of coupling field and power ratio are obtained. The characteristic of coupling field is analyzed. Numerical results show that the target echo will be most obvious and the multipath interference will be weakest when the seeker detects super-low altitude target at the Brewster angle. The further works will focus on studying the characteristics of coupling field from super-low altitude target under complex divisional environments.

\section{REFERENCES}

1. Comite D., Ahmad F., and et al, 2017, Multiview imaging for low-signature target detection in roughsurface clutter environment. IEEE Transactions on Geoscience \& Remote Sensing, 55(9), pp. 52205229.

2. Wang H., Chen Z., and Zheng S., 2017, Preliminary research of low-RCS moving target detection based on Ka-band video SAR. IEEE Geoscience \& Remote Sensing Letters, 14(6), pp. 811-815.

3. Jia C., Guo L., and Liu W., 2017, Parallel FDTD method for EM scattering from a rough surface with a target. IEEE International Symposium on Antennas, Propagation and Em Theory, pp. 569-572.

4. Rashidi-Ranjbar E., and Dehmollaian M., 2015, Target above random rough surface scattering using a parallelized IPO accelerated by MLFMM. IEEE Geoscience \& Remote Sensing Letters, 12(7), pp. 1481-1485.

5. Jonson J. T., 2001, A study of the "four-path" mode for scattering from an object above a half-space. Microwave Optical Technology Letters, 30(2), pp. 130-134.

6. Jonson J. T., 2002, A numerical study of scattering from an object above a rough surface. IEEE Trans. Antennas and Propagation, 50(10), pp. 1361-1367.

7. Zou G. X., Tong C. M., Liu W. M., Chen B., and Wu G. H., 2016, Research on electromagnetic scattering characteristics of strait composite random rough surface based on improved hybrid SBR/PO method. IEEE International Symposium on
Antennas, Propagation and EM Theory, pp. 587591.

8. Zou G. X., Tong C. M., Sun H. L., and et al, 2018, A hybrid method for electromagnetic scattering from target above composite rough surface of ground and near sea in adjacent region. Electromagnetics, 10, pp. 1-23. 